\title{
Multifunctional hypoxia imaging nanoparticles: multifunctional tumor imaging and related guided tumor therapy
}

This article was published in the following Dove Medical Press journal: International Journal of Nanomedicine

Jiajun Liu

Zeying Liu

Daocheng Wu

Key Laboratory of Biomedical Information Engineering of Education Ministry, School of Life Science and Technology, Xi'an Jiaotong University, Xi'an, Shaanxi, China
Correspondence: Daocheng Wu Key Laboratory of Biomedical Information Engineering of Education Ministry, School of Life Science and Technology, Xi'an Jiaotong University, 28 West Xianning Road, Xi'an,

Shaanxi 710049, China

Tel +86298266 394I

Fax +86 298266394 I

Email wudaocheng@mail.xjtu.edu.cn
Abstract: Hypoxia is a common feature of most solid tumors. Having a comprehensive understanding of tumor hypoxia condition is a key to tumor therapy. Many hypoxia imaging nanoparticles have been used for tumor detection. However, simple optical hypoxia imaging is not enough for tumor diagnosis. Also, the tumor therapy process needs the information about the tumor hypoxia condition. Recently, researchers developed multimodal hypoxia tumor imaging nanoparticles and multifunctional hypoxia imaging-guided tumor therapy nanoparticles. The multimodal hypoxia imaging could produce more tumor region information and engage in functional tumor imaging to better understand the tumor condition. The multifunctional hypoxia imaging-guided tumor therapy could monitor the tumor therapy process and evaluate tumor therapeutic effect. Meanwhile, many challenges and limitations are still remaining in the application of multifunctional hypoxia nanoparticles. In this review, we first introduce the types of multifunctional hypoxia imaging nanoparticles. Then we focus on multimodal hypoxia imaging nanoparticles and hypoxia imaging-guided tumor therapy nanoparticles. We also discuss the challenges and future perspectives of this field. There has not been many studies in this field for now. We hope this review would bring more researchers' attention to this field so that it would substantially contribute to tumor precise therapy.

Keywords: nanomedicines, theranostics, hypoxia probe, multimodal tumor imaging, imaging guided tumor therapy

\section{Introduction}

Hypoxia is a physiological or pathological phenomenon related to a wide range of diseases, such as diabetes, ${ }^{1}$ inflammation, ${ }^{2}$ wound, ${ }^{3}$ and tumor. ${ }^{4,5}$ Among these diseases, tumor is a highly dangerous human health problem. The solid tumor has a hypoxic feature. Inside the tumor, cancer cells grow fast. The blood vessels in tumor tissue are fewer than in normal tissue. When the inner oxygen level of a tumor cannot match the needs of the fast-growing cancer cells, a hypoxic region emerges. ${ }^{6}$ The tissues from the outer layer to the inner part of the solid tumor are defined as normoxic, hypoxic, and necrotic $^{6}$ (Scheme 1). Hypoxia can upregulate the hypoxia-induced factor (HIF)-1- $\alpha$ expression. HIF-1- $\alpha$ then upregulates vascular endothelial growth factor (VEGF) and some other downstream proteins to improve blood vessel production and carry further nutrition and oxygen to tumor tissue. ${ }^{4,7}$ Thus, hypoxia becomes a factor that promotes tumor growth and chemo/radiotherapy resistance. ${ }^{8,9}$ Hypoxia is a common feature of most solid tumors. Thus, researchers can use a hypoxia probe to detect tumor and obtain tumor hypoxia distribution images. ${ }^{10-12}$ 


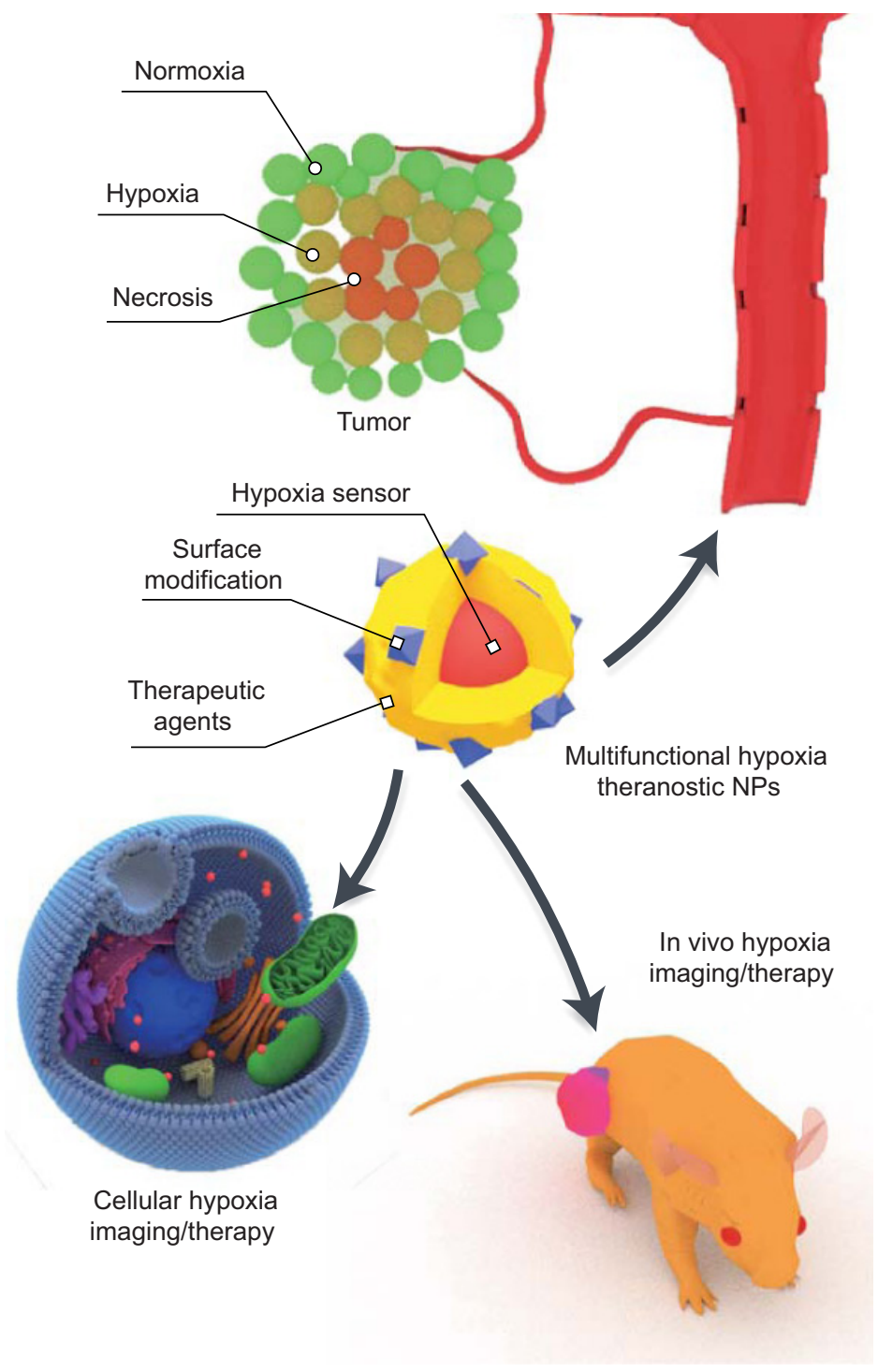

Scheme I The design of multifunctional hypoxia theranostic nanoparticles (NPs).

In recent decades, researchers have greatly contributed to the detection of tumor hypoxia. For clinical diagnosis, the oxygen electrode is the gold standard for oxygen detection. ${ }^{13,14}$ However, this invasive method does not allow imaging. Currently, an increasing number of researchers are focusing on noninvasive hypoxia imaging. The commonly used noninvasive hypoxia imaging modalities are positron emission tomography/computed tomography (PET/CT),${ }^{15}$ magnetic resonance imaging (MRI), ${ }^{16}$ photoacoustic imaging (PAI), ${ }^{17}$ and optical imaging. ${ }^{18}$ Recently, optical hypoxia imaging has gained an increasing amount of attention because of its high sensitivity and output, convenient operation, and low cost. ${ }^{6,19}$ The probes for optical hypoxia imaging are organic dyes ${ }^{20}$ and metal complexes. ${ }^{21,22}$ The metal complex has numerous good features, such as a long phosphorescent life time, high oxygen sensitivity, and lipophilicity to penetrate cell membranes. Thus, this material has become a promising optical hypoxia sensor. The optical hypoxia sensors are mainly applied to cultured cells and animal solid tumor samples. To improve the accuracy and prevent the blocking of tissues and depth influence, researchers developed ratiometric hypoxia sensor, which combines a hypoxic-insensitive dye with a hypoxia sensor and uses their ratio to define the oxygen concentration. ${ }^{18,23}$ Meanwhile, some studies focused on organelle structure hypoxia condition, such as mitochondrial hypoxia distribution. Lv et al developed mitochondrial targeting photosensitizers with hypoxia imaging features. ${ }^{24} \mathrm{By}$ linking the hydrophobic cation moiety, the hypoxia sensor 
achieved a mitochondrial targeting feature and can manifest high-resolution oxygen distribution inside the cell. These new sensors and probes have progressively improved cancer clinical diagnosis.

Hypoxia is a feature that exists throughout tumor genesis and formation. ${ }^{25}$ Solid tumor contains a limited amount of blood vessels. Thus, drugs cannot easily penetrate into the tumor tissue. Moreover, in photodynamic therapy (PDT), hypoxia is a naturally inhibiting condition, because photosensitizers need oxygen to produce singlet oxygen and ROS. ${ }^{26}$ Some researchers found that hypoxia can improve tumor metastasis and invasiveness. ${ }^{27}$ Thus, having a comprehensive understanding of tumor hypoxia condition is key to tumor therapy. Hypoxia imaging can be used to locate areas where tumor can be found. Zheng et al developed an iridiumbased hypoxia nanosensor to detect tumor metastasis. ${ }^{28}$ They used the comicelle method to compose a hypoxia nanosensor (named Ir-CM) from a poly(N-vinylpyrrolidone)-conjugated iridium-(III) complex (Ir-PVP) and poly(e-caprolactone)b-poly(N-vinylpyrrolidone) (PCL-PVP). Under hypoxic conditions, the near-infrared (NIR) phosphorescence of nanosensors is activated. After the intravenous injection of Ir-CM, the cancer metastasis in blood circulation or lymph system can be clearly detected in whole-body imaging.

During therapy, hypoxia imaging can monitor tumor conditions and therapy effects. ${ }^{29}$ At present, tumor hypoxia imaging has been applied in chemotherapy. To monitor tumor microenvironment changes during chemotherapy, Zeng et al used the hypoxia sensor (bis(2-(2'-benzothienyl)-pyridinato$\mathrm{N}, \mathrm{C}^{\prime}$ ) iridium(acetylacetonate)) (BTP, $\left.\operatorname{Ir}(\mathrm{btp})_{2}(\mathrm{acac})\right)$ on CT-26 cell tumor model to monitor tumor hypoxia distribution during cisplatin treatment. ${ }^{11}$ BTP phosphorescent intensity can reflect different cisplatin chemotherapy effects. A larger tumor phosphorescent intensity and smaller tumor volume resulted in a stronger chemotherapy effect. The real-time hypoxia microenvironment in tumor tissue was monitored using the BTP phosphorescence data. The cisplatin chemotherapeutic effect was estimated by phosphorescent intensity and tumor volume data. Thus, hypoxia imaging can be used to monitor and evaluate tumor therapy and for multifunctional hypoxia imaging-guided therapy.

To accomodate all the imaging modalities and tumor therapy drugs with optical hypoxia sensor and to produce a multifunctional hypoxia preparation, the technology requires a carrier upon which all the agents are loaded. A nanoparticle is highly suitable for this purpose. Nanoparticles have suitable features, such as enhanced permeation and retention
(EPR) effect, long circulation, and easy endocytosis, which can help drugs and hypoxia imaging sensors to remain and accumulate in tumor regions. ${ }^{30-32}$ Moreover, the various structural designs of nanoparticles allow each modality and function to work well together without interruption. ${ }^{19}$ By using active targeting and immune escape functional groups, surface modification could also aid the biological application of multifunctional hypoxia nanoparticles. ${ }^{33}$

In this review, we initially introduce the types of multifunctional hypoxia nanoparticles. Then, we focus on multimodal hypoxia imaging nanoparticles and hypoxia imaging-guided tumor therapy. Finally, we discuss the current challenges and future expectations in this field. We hope that this review would attract further research attention to this promising field.

\section{Types of multifunctional and multimodal optical hypoxia imaging and therapeutic nanoparticles}

To combine each modality and functional agent, the nanocarriers should be capable of loading different types of imaging or therapeutic agents. Thus, multifunctional hypoxia imaging nanoparticles have various composite structures. On the basis of structure, the nanoparticles can be classified as follows: core-shell structure nanoparticles, matrix-dispersed nanoparticles, self-assembled nanoparticles, and micelle/ liposome-like nanoparticles. The general descriptions of these composite nanoparticles are listed in Table 1.

\section{Core-shell structure nanoparticles}

Core-shell structure nanoparticles have a core and a shell. These are widely used in studies. Mostly, the core is formatted by a hydrophobic drug or metal complex, which is loaded in a polymer. ${ }^{34}$ Some metal cluster or nanoparticles may also serve as core materials. For the core to become water soluble and biocompatible, the outer shell is usually hydrophilic and composed of biomaterials, such as hyaluronic acid (HA) and chitosan. ${ }^{35}$ The shell enables the core-shell nanoparticles to disperse in water and decreases the cytotoxicity of the core. $\mathrm{Xu}$ et al fabricated a silane coat covering an up-conversion nanoparticle (UCNP) core. Chemotherapy drugs and hypoxia sensors were then loaded into the shell. The UCNP core could convert NIR light into visible light and interact with the drug and sensors in the shell. ${ }^{36}$ Moreover, surface modification adds tumor targeting ability to core-shell nanoparticles. This ability allows nanoparticles to accumulate in the tumor region, thereby benefiting tumor hypoxia imaging and hypoxia imaging-guided tumor therapy. 
Table I Types of multifunctional optical hypoxia imaging nanoparticles

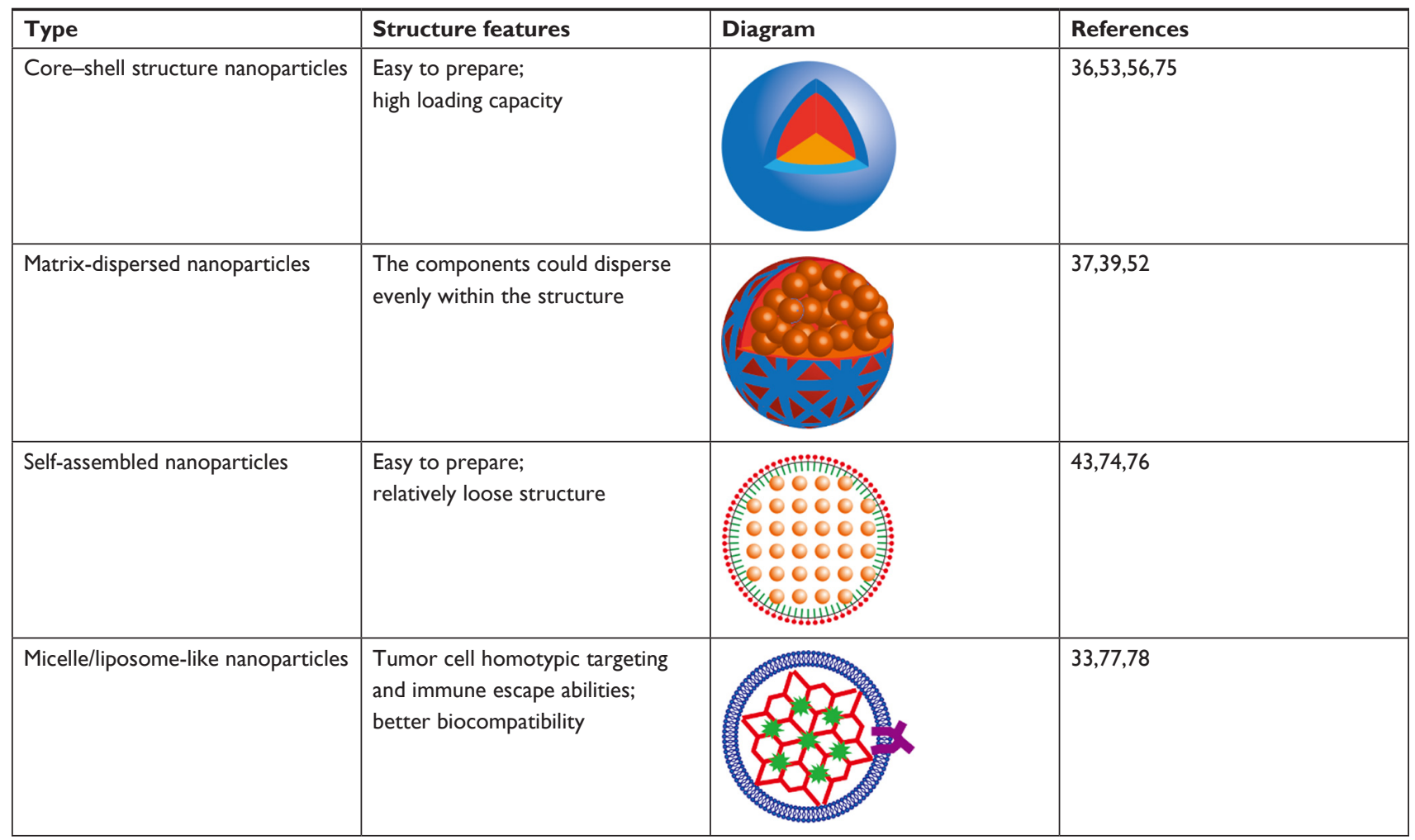

\section{Matrix-dispersed nanoparticles}

The matrix in matrix-dispersed nanoparticles is usually a polymer. The hypoxia probe, imaging agent, and tumor therapy agent are dispersed evenly in the matrix. Those agents could be small molecules and small nanoparticles. Huang et $\mathrm{a}^{37}$ developed supramolecular polymer nanoparticles (SPNPs) for ratiometric tumor hypoxia imaging and therapy. Pt(II) porphyrin for hypoxia imaging and PDT, and the hypoxia-insensitive reference dye 9,10-diphenylanthracene were distributed in the SPNPs. ${ }^{37}$ In some cases, small nanoparticles were encapsulated into the matrix to become larger nanoparticles. ${ }^{38}$ The small nanoparticles are metal clusters, quantum dots (QDs) and UCNP, serving as fluorescent functional agents. The matrix separates the small nanoparticles and protects from self-quenching and other interactions. After being loaded in nanoparticles and matirx, the hypoxia probes, fluorescent functional agents, or therapy agents will interact with each other to realize the multifunctional hypoxia imaging. These agents can be mixed more thoroughly in one matrix-dispersed nanoparticle structure than in a simple core-shell structure, in which only the materials near the edge are close to one another. $\mathrm{Yu}$ et al loaded metal-enhanced fluorescent (MEF) agent gold nanoparticles (Au NPs) and hypoxia sensor BTP into a polyiohexol matrix. ${ }^{39}$ The Au NPs and BTP were thoroughly mixed to allow the Au NP luminescence to fully enhance the BTP luminescent signal.

\section{Self-assembled nanoparticles}

Self-assembled nanoparticles use amphiphilic block polymers to format a nanoparticle. ${ }^{40}$ The core usually comprises hydrophobic agents. However, unlike core-shell nanoparticles, the block polymer and core interaction of self-assembled nanoparticles are induced in one step. ${ }^{41}$ Self-assembled nanoparticles usually need fewer kinds of agents to compose nanoparticles. Thus, the biocompatibility of self-assembled nanoparticles is much better than those of the other kinds of nanoparticles. ${ }^{42}$ Zhou et al prepared self-assembled hypoxia imaging and tumor PDT nanoparticles by using Pt(II) porphyrin and polyfluorene-based hyperbranched conjugated polyelectrolyte. The materials self-assembled into ultrasmall polymer dots (Pdots) $\sim 10$ $\mathrm{nm}$ in size. ${ }^{43}$ The loading capacity of self-assembled carriers are usually not as large as that of normal nanocarriers. The polarity and hydrophobicity of hypoxia sensor molecules and drugs should be considered in designs. Self-assembled nanoparticles are relatively loose in structure and are only restrained by van der Waals forces. Some researchers use 
cross links to further solidify their nanoparticles after the self-assembly process.

\section{Micelle/liposome-like nanoparticles}

Micelles and liposomes have been widely used in drug delivery and emulsion preparation. ${ }^{44}$ These structures remain very good nanocarriers for optical hypoxia sensors and drugs. Micelles have a single layer for loading lipophilic agents and liposomes have double layers that can be loaded inside with a hydrophilic water phase. Meanwhile, the lipid phase can still be mixed with some lipophilic agents. In recent years, some studies exploited cell membranes, such as the cancer cell membrane, to realize homotypic targeting and immune escape abilities, for tumor targeting. ${ }^{45} \mathrm{Li}$ et al applied cancer cell membranes on Pt(II) porphyrinic metal-organic framework (PPt) to prepare membrane@Pt(II) porphyrinic metal-organic framework (mPPt). The mPPt has a distinctive tumor targeting feature..$^{33}$ The materials used in preparing micelles and liposomes are phospholipids, amphiphilic polymers, and cell membranes. Thus, their biocompatibilities are much better than those of other composite nanoparticles.

\section{Multimodal hypoxia imaging nanoparticles}

Although progress has been achieved in cancer diagnosis techniques, numerous patients notice their cancer condition only at a late stage. Early-stage cancer detection allows doctors and patients to pay attention to subclinical cancer and to address the condition immediately before it progresses and metastasizes. Hypoxia is an ideal parameter that distinguishes tumor tissue from normal tissue. ${ }^{6}$ Hypoxia is an event that contributes to the entire tumor growth process. The HIF and VEGF pathways need hypoxia conditions to upregulate and then improve tumor growth. Thus, thoroughly understanding the tumor micro hypoxia environment can help improve cancer therapeutic methods. Using an effective hypoxia sensor to detect early stage tumor and image the tumor hypoxia distribution can profoundly contribute to cancer therapy. Optical hypoxia imaging is a fast and convenient way to achieve overall hypoxia distribution and tumor detection.

Optical hypoxia imaging is a high-sensitivity imaging method. However, the resolution of in vivo fluorescent/ phosphorescent imaging is relatively low compared with those of high-resolution imaging modalities, such as MRI, $\mathrm{CT}$, and PAI ${ }^{46}$ Hence, the combination of high-resolution imaging modalities and high-sensitivity functional hypoxia imaging make this method valuable for detecting tumor and for thoroughly understanding inner tumor conditions. ${ }^{30}$
CT is a widely used clinical imaging method. CT produces high-resolution and 3D images. Optical hypoxia imaging is a highly sensitive functional imaging method to reflect hypoxia distribution. The combination of CT and optical hypoxia imaging is a better way to detect tumor hypoxia distribution with high-resolution morphology image. Yu et al developed a dual modal-enhanced phosphorescence hypoxia/CT imaging nanoprobe based on iridium complex BTP, $\operatorname{Ir}(\mathrm{btp})_{2}(\mathrm{acac})$ and Au NPs polyiohexol composite (BAPI) nanoparticles. ${ }^{39}$ The scholars used polyiohexol to encapsulate Au NPs and BTP. $\mathrm{Au}$ NPs and polyiohexol served as CT imaging agent, and BTP acted as a hypoxia sensor. The BAPI nanoparticles had a size distribution of $52.9 \mathrm{~nm}$. On a hela cell subcutaneous tumor model, the CT imaging results showed high-quality 3D CT images. The BAPI nanoparticles exhibited a high CT value of 800 , which is higher than that of the commercial CT agent iohexol (400). Au NPs can also be used as an MEF agent. The phosphorescent intensity of BTP was increased eightfold in vitro by the Au NPs. When hypoxia imaging was performed in vivo, the BAPI nanoparticles accumulated in the tumor area from 24 to $48 \mathrm{~h}$ post-injection, and the hypoxia distribution of the tumor is observed in photographs. The phosphorescent intensity of BAPI in tumor was higher than that of the BPI nanoparticles and BTP solution, thereby indicating the MEF effect of the Au NPs (Figure 1A).

Jana et al prepared dual modal MRI/hypoxia imaging probes based on iridium (III)-lanthanide (III) luminescent complexes. ${ }^{47}$ The authors successfully connected a phosphorescent iridium (Ir) unit with one or two lanthanide (Ln, $\mathrm{Ln}=\mathrm{Eu}(\mathrm{III})$ and $\mathrm{Gd}(\mathrm{III})$, respectively) units to synthesize an Ir-Ln metal complex for MRI/luminescent bioimaging. The dinuclear complex had outstanding water solubility, low cytotoxicity, and accurate lysosome staining ability. For dual modal imaging, the Ir-Gd complex has a very high reflexivity (11.9 $\mathrm{mM}^{-1} \mathrm{~s}^{-1}$ in water) compared with monoGd complexes, such as diethylenetriaminepentaacetic acid and 1,4,7,10-tetraazacyclododecane-1,4,7,10-tetraacetic acid (4-5 $\mathrm{mM}^{-1} \mathrm{~s}^{-1}$ ). The two-photon phosphorescence lifetime imaging (PLIM) experiment showed excellent $\mathrm{O}_{2}$-sensing ability in vitro of the Ir-Gd complex (Figure 1B).

\section{Hypoxia imaging-guided tumor therapy nanoparticles}

Numerous multifunctional image-guided cancer therapy nanoparticles have been developed to build a theranostic platform for cancer treatment. In a traditional cancer therapeutic process, hypoxia constantly acted as a hindering factor 


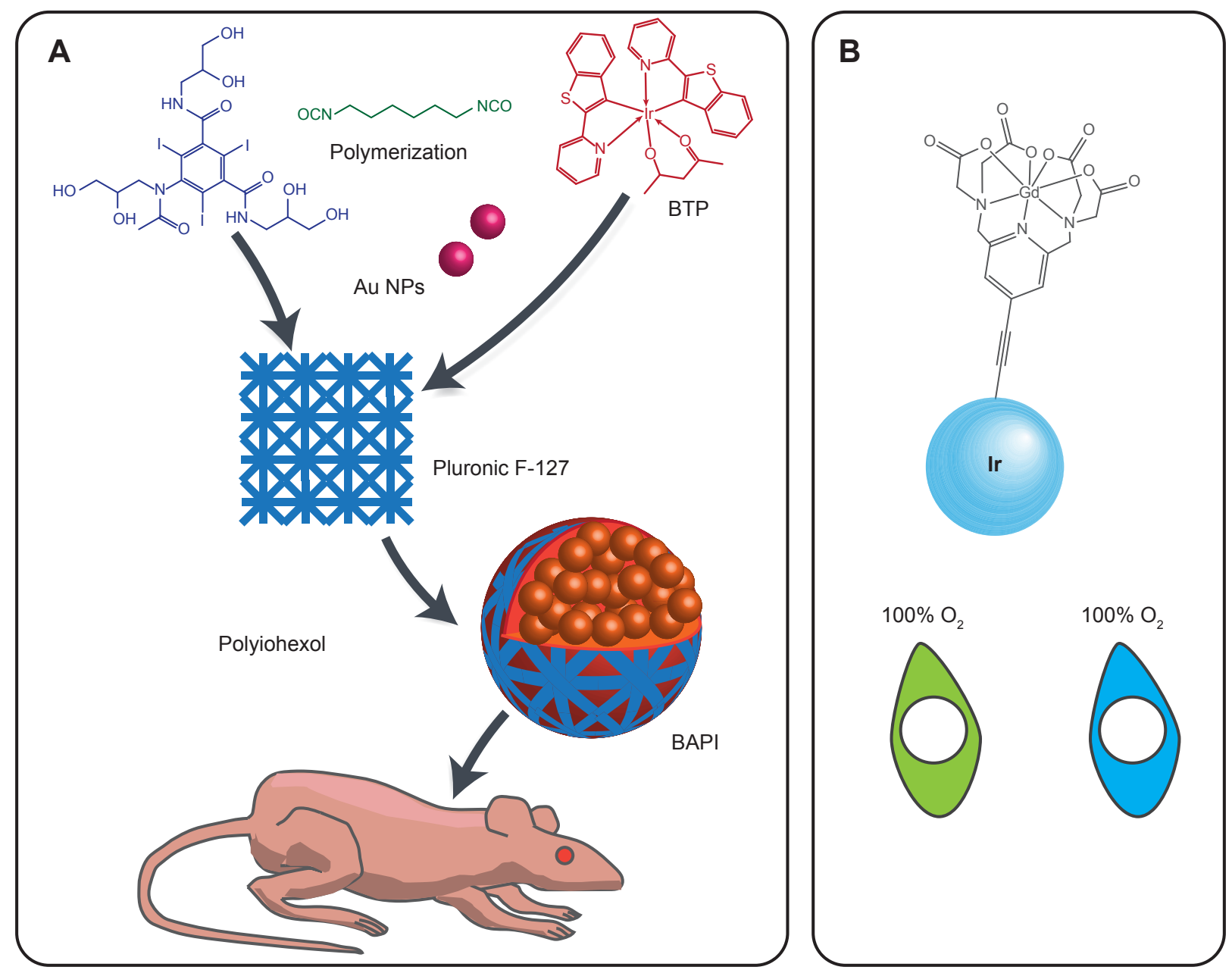

Figure I A schematic representation of the synthetic process of Au NP polyiohexol composite BAPI nanoparticles and the dual-model imaging of BAPI nanoparticles (A). A schematic illustration for the Ir-Gd complex and its application for optical hypoxia/MRI dual modal imaging (B).

Abbreviations: Au NP, gold nanoparticle; BTP, Ir(btp) ${ }_{2}$ (acac); btp, 2-(2'-benzothienyl)-pyridinato-N,C3'; acac, acetylacetonate; BAPI, BTP and gold nanoparticles polyiohexol composite; MRI, magnetic resonance imaging.

for doctors and patients. This condition made solid tumor resistant to chemotherapy and radiotherapy. Given the insufficiency of blood vessels, the solid tumor is almost out of normal blood circulation. Thus, few cancer therapy drugs can reach the tumor area. Insufficient oxygen supply is also adverse to PDT. Hypoxia can trigger cancer cell mutation and tumor metastasis. Therefore, understanding the tumor hypoxic degree and internal tumor hypoxia distribution can help address hypoxia in tumors. The hypoxia imaging method can aid in locating the tumor area. To further utilize optical hypoxia imaging to guide cancer therapy and monitor therapeutic efficiency, researchers built various theranostic systems for optical hypoxia imaging-guided tumor therapy.

\section{Hypoxia imaging-guided PDT}

Many phosphorescent hypoxia probes can produce ROS, which can eliminate cancer cells. ${ }^{48}$ This kind of therapeutic method is called PDT. These ROS-generating agents are called photosensitizers. PDT is a promising cancer therapy method that has a better therapeutic effect than chemotherapy on chemotherapy-resistant tumor types. ${ }^{49}$ Chemotherapy has its drawbacks, such as side effect, drug resistance, and toxicity to normal organs and cells. PDT is a noninvasive therapy method with high space accuracy. It is an imagingguided therapy method.

PDT uses cytotoxic singlet oxygen species and ROS to treat cancer cells. The triplet oxygen molecules react with photosensitizers and then transform into singlet oxygen species. PDT has a high therapy efficiency and high area selectivity. PDT needs light source irradiation; thus, the therapy selectivity can be controlled by carefully applying a light source to the cancer area. Under radiation, the photosensitizer converts oxygen to singlet oxygen-free radicals, which kill cancer cells. The singlet oxygen has a limited half-life and space diffusion rate. The photosensitizer is nontoxic without light irradiation, thereby preventing damage to normal tissues. 
The phosphorescent hypoxia probes use a similar mechanism to react with oxygen molecules. The probes are excited from the singlet state transition to the triplet state by intersystem crossing process. Then, the phosphorescence is quenched by oxygen molecules. Oxygen sensing is based on the linear relationship between the phosphorescent intensity change and the oxygen concentration. ${ }^{50}$ Thus, these two process can be integrated together as hypoxia imaging-guided PDT. Light, oxygen, and photosensitizer are the three main factors in PDT. ${ }^{51}$ The hypoxia in tumor microenvironment can lead to tumors' PDT resistance. Thus, the investigation of the inner tumor hypoxia distribution is much more important in accurately diagnosing and evaluating the tumor PDT effect.

Therefore, building a multifunctional nanoparticle for tumor hypoxia distribution sensing while retaining the photosensitizer feature in situ is very useful. This technology is highly effective in mapping early stage tumor and killing cancer cells by light irradiation. Feng et al prepared Ir(III) complex hyperbranched phosphorescent-conjugated Pdots for hypoxia imaging and PDT. ${ }^{52}$ The scholars chose phosphorescent oxygen sensor fac-tris(1-phenylisoquinolinato-N,C2')iridium(III) and the oxygen-insensitive dye 9,9-dioctylfluorene to synthesize hyperbranched conjugated polymer (Ir-HPC). The surface was coated with poly(styreneco-maleic anhydride) (PSMA) to fabricate Ir-HPC/PSMA dots. The PSMA coating enhanced the water solubility and brought negative charge to Ir-HPC/PSMA dots. The Ir oxygen sensor then achieved great oxygen sensing ability. The Förster resonance energy transfer (FRET) between the 9,9-dioctylfluorene and Ir sensor resulted in Ir-HPC/ PSMA dot ratiometric oxygen sensor. Ir-HPC/PSMA dots can be used on PLIM and time-gated luminescence imaging to decrease autofluorescence and background noise. The Ir-HPC/PSMA dots had high ROS yield. The hypoxia image-guided in situ PDT results were confirmed by confocal microscopy and MTT assay. Cell death and apoptosis can be observed in situ.

The same work was performed by Zhou et $a 1 .^{43}$ They prepared hypoxia imaging-guided cancer cell PDT multifunctional phosphorescent-conjugated Pdots. ${ }^{43}$ The scholars also used Pt(II) porphyrin as an oxygen sensor and polyfluorene-based hyperbranched conjugated polyelectrolyte as a reference. These materials can be self-assembled into $\sim 10 \mathrm{~nm}$ Pdots. These Pdots improve the water solubility of Pt porphyrin. The FRET between the Pt complex and polyfluorene resulted in nanosensor ratiometric oxygen sensing. For PDT efficiency, the Pdots obtained a high ROS quantum yield of 0.80 in solution. The MTT assay showed excellent cancer cell ablation ability. The flow cytometry and real-time fluorescent microscopy results also indicated superior PDT efficiency.

Wang et al synthesized mitochondria targeting nanoparticles for the optical hypoxia imaging and PDT..$^{53}$ They doped Pt(II)-meso-tetra(pentafluorophenyl)porphine (PtTFPP) into nanoparticles for oxygen sensing and PDT. The scholars also modified the nanoparticles with mitochondrial targeting agent triphenylphosphine. Under low-energy irradiation, the nanoparticle serves as an oxygen sensor to monitor respiratory activities. Under high-energy irradiation, the nanoparticles can generate ROS for PDT to treat cancer cells. The cytotoxicity assay and oxygen consumption rate assay showed excellent PDT efficiency (Figure 2A).

The cell-level effect of PDT is very distinct. However, the final goal of PDT is to treat cancer. Li et al developed a biomimetic theranostic $\mathrm{O}_{2}$ meter for tumor targeting PDT and optical hypoxia imaging. ${ }^{33}$ The scientists coated $\mathrm{mPPt}$ for tumor targeting and immune escape. The mPPt can monitor real-time tumor oxygen distribution and fluctuation in vivo. The mPPt also showed excellent tumor targeting ability. The anticancer study showed that the mPPt and PPt groups achieved a slightly reduced tumor size relative to those of the control groups and indicated the satisfactory PDT effect of $\mathrm{mPPt}$ (Figure 2B).

Lv et al developed a multifunctional hypoxia imaging probe based on phosphorescent starburst Pt(II) porphyrins for tumor hypoxia imaging and PDT. ${ }^{54}$ The group synthesized a couple of hydrophilic starburst Pt(II) porphyrins with cationic fluorene oligomers as arms. These arms rendered these starbursts hydrophilic and provided a 3D structure to prevent aggregation. Longer arms resulted in superior oxygen sensitivity and ROS generation ability. Among the agents, one of the hydrophilic starburst Pt(II) porphyrins (Pt-3) had a deep-red emission and long phosphorescent lifetime, thereby eliminating autofluorescence and increasing the accuracy of oxygen sensing. The in vitro PDT effect was confirmed by MTT assay and flow cytometry. The Pt-3 still had higher PDT efficiency than that of hematoporphyrin (HP, a typical photosensitizer in clinic) even under hypoxic conditions. In vivo tumor inhibition experiments showed that Pt-3 had better PDT efficiency than HP (Figure 2D).

\section{Hypoxia imaging-guided chemotherapy}

The direct inhibition of HIF is an effective way to treat hypoxia-associated diseases. Combining hypoxia imaging and HIF inhibition is an alternative strategy for hypoxia imaging-guided cancer therapy. Wang et al prepared a ruthenium(II) anthraquinone complex-based hypoxia imaging 

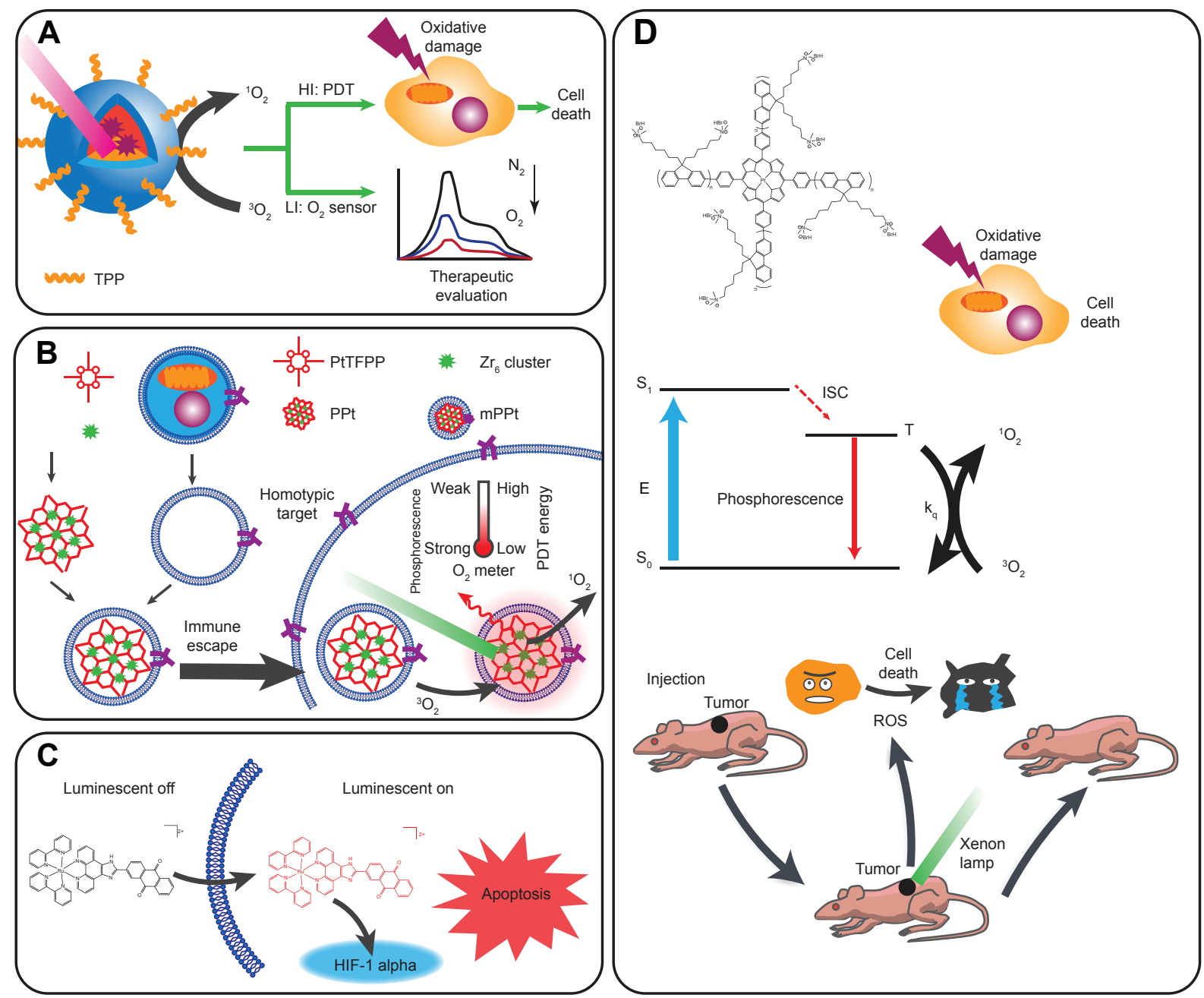

Figure 2 A schematic illustration of PtTFPP-doped nanoparticle and TPP modification and its application in optical hypoxia sensing and hypoxia imaging-guided PDT (A). A schematic illustration of mPPt synthesis and cell membrane coating and its application in PDT and hypoxia imaging (B). A schematic illustration of Ru-ipad chemical structure and its application in optical hypoxia sensing and inducing apoptosis (C). A schematic illustration for the synthesis of Pt complex probe and its oxygen-sensing mechanism and its application in tumor PDT (D).

Abbreviations: HI, high energy irradiation; LI, low energy irradiation; TPP, triphenylphosphine; HIF, hypoxia-induced factor; mPPT, membrane@Pt(II) porphyrinic metalorganic framework; PDT, photodynamic therapy; PtTFPP, Pt(II)-meso-tetra(pentafluorophenyl)porphine; ROS, reactive oxygen species; Ru-ipad, Ru(bpy)2(ipad)](CIO4)2; bpy, 2,2'-bipyridine; ipad, 2-(anthracene-9, I0-dione-2-yl)imidazo[4,5-f] [I,I0]phenanthroline).

agent combined HIF-1- $\alpha$ inhibition. ${ }^{55}$ They prepared $\left[\mathrm{Ru}(\mathrm{bpy})_{2}(\mathrm{ipad})\right]\left(\mathrm{ClO}_{4}\right)_{2}\left(\mathrm{Ru}\right.$-ipad, where bpy $=2,2^{\prime}$-bipyridine and ipad $=2$-(anthracene-9,10-dione-2-yl)imidazo[4,5-f] $[1,10]$ phenanthroline) for hypoxia sensing and cancer inhibition. The Ru-ipad had a 40-fold phosphorescent intensity change from $20 \%$ to $1 \%$ oxygen level. When applied to cultured cells, Ru-ipad showed excellent intracellular hypoxia imaging ability. The Ru-ipad also exhibited cytotoxicity in $2 \mathrm{D}$ and $3 \mathrm{D}$ cultured cells. The MTT assay showed that the $\mathrm{Ru}$-ipad had a similar $\mathrm{IC}_{50}$ value as that of commercial clinical chemotherapy drug cisplatin at around $20 \mu \mathrm{M}$. In cisplatinresistant cell lines, $\mathrm{Ru}$-ipad still has a low $\mathrm{IC}_{50}$, whereas cisplatin's $\mathrm{IC}_{50}$ reached $\sim 150 \mu \mathrm{M}$. In the 3D-cultured tumor sphere model, Ru-ipad manifested tumor inhibition within $0-8$ days. The tumor sphere volume showed no change compared with the control group, which was similar to the cisplatin group. Ru-ipad could inhibit HIF-1- $\alpha$ expression to treat cancer cells under hypoxic conditions (Figure 2C).

Li et al developed an up-converting NIR irradiation/ hypoxia responsive nanotheranostic agent for hypoxia imaging and tumor therapy. ${ }^{56}$ The nanoparticle is based on UCNP. A novel anticancer drug floxuridine (FDU) was modified by an ultraviolet photo trigger (E)-o-hydroxycinnamic acid $\left(\mathrm{CA}_{\mathrm{E}}\right)$ and hypoxic trigger 4-nitrobenzyl group. Then they were all linked to UCNP to be a nanotheranostic platform (designed as UCNP-CA - EDU/NO $\mathrm{NO}_{2}$ ). When treated with $980 \mathrm{~nm}$ light and under hypoxic conditions, the nanoparticle UCNP-CA - EDU/NO $\mathrm{NO}_{2}$ can release FDU to treat tumor and emit fluorescence for hypoxia imaging. The cellular imaging of the cell culture showed nearly tenfold 
increase of the fluorescent intensity of UCNP-CA $-\mathrm{EDU} /$ $\mathrm{NO}_{2}$ after incubation with oxygen levels from $20 \%$ to $1 \%$. In vivo tumor inhibition experiment was performed with the MCF-7 cell line tumor model. The mouse group injected with UCNP-CA $A_{\mathrm{E}}-\mathrm{FDU} / \mathrm{NO}_{2}$ and treated with NIR radiation had excellent tumor inhibition rates $(86 \%)$ compared with the control group after 14 days. Meanwhile, the NIR treatment only and nanoparticle injection only groups showed lower tumor inhibition and could only maintain the tumor growth and not decrease the tumor size as in the nanoparticle-combined NIR group (Figure 3A).

Qian et al prepared a light-activated hypoxia-responsive nanocarriers for hypoxia imaging and tumor PDT/ chemotherapy. ${ }^{43}$ The group combined PDT and chemotherapy into a light-activated and hypoxia-responsive doxorubicin hydrochloride hypoxia-sensitive 2-nitroimidazole-grafted conjugated polymer nanoparticles (DOX/CP-NI NPs). The DOX/CP-NI NPs had a good PDT and drug effect in vitro. The DOX/CP-NI NPs can accumulate in tumor areas after intravenous injection. The tumor growth was completely inhibited in the DOX/CP-NI NPs and light-treated groups
5 days after injection compared with those in the doxorubicin hydrochloride only, PDT only, DOX/CP-NI NPs without light, light only, and saline control groups. This result indicated the higher combined therapeutic efficacy of doxorubicin hydrochloride and PDT.

\section{Challenges and future perspectives}

The multifunctional hypoxia imaging and therapy nanoparticles have many advantages in tumor detection and imaging-guided tumor therapy. However, some limitations still limit the application of this technology. Multifunctional and multimodal nanoparticle-based theranostic system for treating tumor has numerous differences relative to those of traditional cancer imaging and therapeutic methods. The multifunctional hypoxia nanoparticle has more constituents and complicated structures. Thus, the interruptions between hypoxia sensors and other luminescent dyes or therapeutic drugs have become a problem. Additional intervals must be built to separate agents and maintain their functions. Thus, a further complicated structure introduces challenges to the quality control of the nanoparticle synthesizing process.
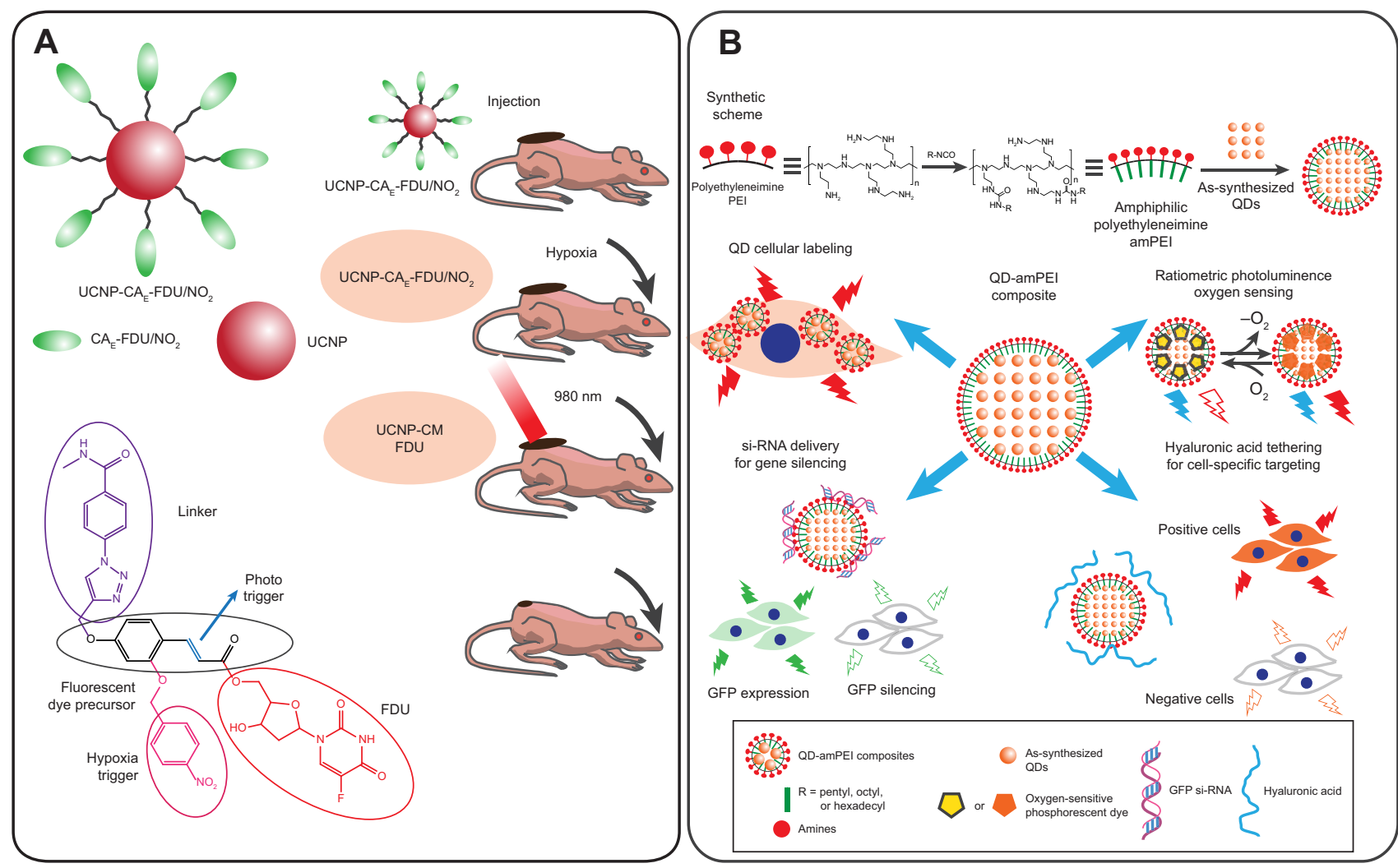

Figure 3 A schematic illustration of the UCNP-CA $-F D U / N_{2}$ structure of the mechanism of hypoxia/NIR activating for tumor imaging and imaging-guided tumor therapy $(\mathbf{A})$. A schematic representation for the amPEI synthesis process, and the application of amPEl in cell labeling, gene delivery, cancer cell targeting, and ratiometric hypoxia sensing (B).

Abbreviations: amPEI, amphiphilic polyethyleneimine; $\mathrm{CA}_{\mathrm{E}}$, (E)-o-hydroxycinnamic acid; FDU, floxuridine; GFP, green fluorescent protein; NIR, near-infrared; QDs, quantum dots; UCNP, up-conversion nanoparticle. 
Meanwhile, the more imaging modalities or therapy methods are involved, the more complicated the nanoparticle becomes and the more difficult for researchers to coordinate each component. The current multimodal hypoxia imaging nanoparticles usually have only one modality with a distinguishing performance; the other modalities were not as good but usually served as add-on morphology function, which is still not superior. All the functions are supposed to cooperate with one another and achieve a synergistic effect. Optical hypoxia imaging is an ideal imaging modality that can match other imaging and therapy methods. The high sensitivity can combine with the high-spatial-resolution methods, such as CT and PAI. Hypoxia-monitoring ability is important to cancer therapy. To build more robust multifunctional hypoxia imaging and therapy nanoparticles, the imaging performance of each modality need to be improved to fulfill the requirements for hypoxia sensing, referring, and functional imaging.

Moreover, the imaging modalities selected should consider other tumor biology factors besides simply recreating the morphology of the tumor region. Multimodal hypoxia tumor imaging is intended for tumor therapy. Knowing the shape and location of tumor is obviously not enough. Hypoxia imaging can detect tumor and show the hypoxia distribution condition within the tumor region. Meanwhile, this modality not only guides tumor therapy but also monitors the therapy process and contributes to decision making. ${ }^{57}$ Thus, the modalities involved in multifunctional hypoxia imaging nanoparticles may include biology parameters, such as tumor $\mathrm{pH}$ imaging, temperature sensing, and cancer cell extracellular biomarker detection.

To achieve tumor hypoxia imaging, the nanoparticle should first penetrate into the tumor area. Nanoparticles first go through blood vessels to reach the tumor region. The current nanoparticles with a diameter around $100 \mathrm{~nm}$ can only be accumulated in solid tumor through EPR effect. However, during the process of nanoparticles transferring from the vascular to tumor hypoxic tissues, there are still many collagens, glycosaminoglycans, and proteoglycans blocking the nanoparticles from spreading throughout the entire tumor. ${ }^{58}$ The normal drug delivery in tumor extracellular matrix $(\mathrm{ECM})$ went through passive diffusion process, which depends on the interstitial fluid pressure. However, for large particles, the passive diffusive transport is not enough. The ECM is composed of different materials such as collagen, glycosaminoglycans, and proteoglycans..$^{59}$ The complex mixture reduces the spread rate of nanoparticles. Among these compositions, collagen plays an important role in hindering the diffusion of nanoparticles. The distance between collagen fibers is $20-40 \mathrm{~nm}$. The collagen bubble space between
75 and $130 \mathrm{~nm}$ would also reduce the delivery of nanoparticles. ${ }^{60}$ Thus, in the design of multifunctional hypoxia imaging nanoparticles, the particle size that could match the ECM limitation window becomes a crucial factor to be considered. The multifunctional hypoxia imaging nanoparticles' size distribution is better if $<40 \mathrm{~nm}$. Another strategy is to first produce large nanoparticles with a diameter around $100 \mathrm{~nm}$. When triggered by the environmental parameters, they shrunk into smaller nanoparticles to penetrate the tumor ECM. ${ }^{61} \mathrm{Xie}$ et al prepared hypoxia-induced size-shrinkable nanoparticle to improve the nanoparticle penetration in tumor hypoxia region. ${ }^{62}$ Both the designs would assist the distribution of the multifunctional hypoxia nanoparticles in solid tumor and produce more reliable hypoxia imaging data.

The nanoparticle carrier can improve the effects of tumor hypoxia detection and hypoxia imaging-guided tumor therapy. The proteins and peptide drugs are macromolecules with EPR effect. ${ }^{63}$ Some peptide drugs with active targeting capabilities could also target tumor region. ${ }^{64}$ Researchers have used these peptides and proteins as drug carrier or probe. Zhou et al prepared bombesin-conjugated ${ }^{111}$ In complex for hypoxia-enhanced prostate cancer imaging. ${ }^{65}$ However, proteins and peptides have some drawbacks that limit their performance in doing hypoxia imaging. They are vulnerable to enzyme degradation. The mononuclear phagocyte could also uptake those peptides. The renal filtration also decreases the circulation time of peptides. ${ }^{66,67}$ The nanoparticles that carry small molecules could accumulate in the tumor area by EPR effect and then release those small molecules to be defused in tumor tissue. The nanoparticle-based hypoxia imaging probe has longer circulation time than peptides and protein probes. ${ }^{68}$ They are not likely to be cleared by renal filtration. ${ }^{69}$ The surface modification could add stealth materials such as polyethylene glycol to prevent nanoparticles from being taken up by mononuclear phagocyte. ${ }^{70}$

After the nanoparticles reach the tumor hypoxia tissue, the next step is to receive the signals from deep tumor tissue. The autofluorescent absorption from the body and decay of optical signals render many kinds of optical imaging methods not applicable to tumor sampling deep in the tissues. ${ }^{71}$ The same scenario was observed in PDT. At present, the in vivo experiments performed in optical hypoxia imaging-related papers mostly involved subcutaneous tumor models. A design incorporating the use of NIR dye can improve the transmission of fluorescent signals. The NIR window of biological tissue, that is, between 650 and $1,350 \mathrm{~nm}$, allows fluorescence to transmit more strongly than the other wavelength ranges. ${ }^{72}$ NIR hypoxia imaging has a stronger signal and offers more accurate results than 
those of other modalities. Optical hypoxia sensors must be developed and applied to improve penetration into tissues. The current up-conversion hypoxia imaging nanoparticle is a good way to partially solve this issue because of its use of NIR excitation instead of visible light. ${ }^{73}$ However, NIR emissive hypoxia sensors and photosensitizers are still crucial for realizing multifunctional hypoxia nanoparticle clinical application.

Another limitation is the need for a standard monitoring rule system for hypoxia imaging-guided tumor therapy. The tumor precise therapy requires not only to show the morphology of the tumor site but also to monitor and to evaluate the therapy effect. Thus, an accurate system evaluating the cancer therapeutic effect that is based on the hypoxia distribution variation within the tumor area is crucial to hypoxia imaging-guided cancer therapy. Current hypoxia imaging-guided therapy usually involves hypoxia imaging as the only imaging modality. However, hypoxia signal alone is insufficient for precise evaluation because of the tissue block and autofluorescence that affect the luminescent intensity. Ratiometric hypoxia imaging can quantify oxygen concentration based on luminescent intensity. Meanwhile, hypoxia monitoring still entails additional parameters, such as the tumor size/volume, and another imaging modality to achieve accuracy. In this case, the multimodal hypoxia imaging-guided tumor therapy is a more rational design. Current imaging or therapy is still not well integrated with the hypoxia sensor.

Currently, the optical hypoxia imaging-guided tumor therapy mainly includes PDT and chemotherapy. The optical hypoxia imaging-guided PDT has been well developed since the PDT process needs to monitor the tumor oxygen condition in order to enhance PDT effect. The chemotherapeutic effect could also be evaluated by optical hypoxia imaging. In recent years, tumor gene therapy has become a promising way to treat tumors. Some researchers have already applied hypoxia imaging to guide the tumor gene therapy. Park et al developed a multifunctional nanoparticle platform based on amphiphilic polyethyleneimine (amPEI), tris(4,7-diphenyl1,10-phenanthroline)ruthenium(II) $\left(\mathrm{Ru}(\mathrm{dpp})_{3}{ }^{2+}\right)$ hypoxia probe and QDs for cellular labeling, targeting, gene delivery, and ratiometric oxygen sensing. The authors prepared QD and $\mathrm{Ru}(\mathrm{dpp})_{3}{ }^{2+}$ cohosting amPEI composite (QD-Ru-amPEI) as multifunctional nanoparticles for cellular labeling, hypoxia sensing, and gene delivery. ${ }^{74}$ QD-Ru-amPEI had excellent ratiometric oxygen-imaging results on HCT-116 cells. The tumor sphere model showed a 3D hypoxia distribution after application with QD-Ru-amPEI. The amPEI loaded with small interfering RNA can silence green fluorescent protein gene expression in cells. When coated with HA, amPEI showed active cancer cell-targeting ability (Figure 3B). The hypoxia imaging can monitor the tumor hypoxia condition during the gene therapy along with evaluating the gene therapeutic effect. The multifunctional hypoxia imaging and gene therapy nanoparticles have the potential to be applied in tumor gene therapy.

In summary, the current multifunctional hypoxia nanoparticle is a promising technology for tumor detection and therapy, although it is currently facing some drawbacks and limitations. Several of its features, such as sensitivity, biocompatibility, and therapeutic efficiency, can still be improved. The other biological parameter sensors combined with hypoxia imaging are also a potential progressive point. To date, not many tumor-related studies have explored hypoxia. Hypoxia is a crucial factor for tumor detection and tumor therapeutic effect evaluation. With future progress, this technology will eventually become a powerful way to satisfy the need for tumor diagnosis and therapy.

\section{Conclusion}

Precise tumor therapy requires technological progress in tumor imaging and therapy. Hypoxia is a key factor involved in the whole tumor genesis, growth, metastasis, and invasiveness processes. Investigating hypoxia distribution and understanding the hypoxia condition in tumor areas can greatly contribute to cancer diagnosis and therapy. The current multifunctional hypoxia nanoparticles have numerous advantages and have achieved great progress in the detection of tumor and the guidance of tumor therapy in the laboratory. Multimodal hypoxia imaging helps rapidly locate tumor regions non-invasively and obtain functional images of tumor areas. Hypoxia imaging-guided tumor therapy can monitor the therapy process and evaluate the therapeutic efficiency. However, many challenges remain in the clinical application of this technology. The issues of signal transmission, therapeutic effect estimating standard, and the combination of multimodal hypoxia imaging need to be addressed. Overall, the multifunctional hypoxia nanoparticle is a promising and powerful tool for treating tumor, and multimodal optical hypoxia imaging. Hypoxia-guided tumor therapy can substantially contribute to precise tumor theranostic.

\section{Acknowledgments}

This work was sponsored in part by the National Natural Science Foundation of China (Nos 81471771 and 81871476) and the National Key Scientific Instrument and Equipment Development Project of China (No 81827801). 


\section{Disclosure}

The authors report no conflicts of interest in this work.

\section{References}

1. Azevedo JL, Carey JO, Pories WJ, Morris PG, Dohm GL. Hypoxia stimulates glucose transport in insulin-resistant human skeletal muscle. Diabetes. 1995;44(6):695-698.

2. Eltzschig HK, Carmeliet P. Hypoxia and inflammation. $N$ Engl J Med. 2011;364(7):656-665.

3. Allen DB, Maguire JJ, Mahdavian M, et al. Wound hypoxia and acidosis limit neutrophil bacterial killing mechanisms. Arch Surg. 1997; 132(9):991-996.

4. Maxwell PH, Dachs GU, Gleadle JM, et al. Hypoxia-inducible factor-1 modulates gene expression in solid tumors and influences both angiogenesis and tumor growth. Proc Natl Acad Sci U S A. 1997; 94(15):8104-8109.

5. Höckel M, Vaupel P. Tumor hypoxia: definitions and current clinical, biologic, and molecular aspects. J Natl Cancer Inst. 2001;93(4): 266-276.

6. Liu JN, Bu W, Shi J. Chemical design and synthesis of functionalized probes for imaging and treating tumor hypoxia. Chem Rev. 2017;117(9): 6160-6224.

7. Ryan HE, Poloni M, Mcnulty W, et al. Hypoxia-inducible factor-1 $\alpha$ is a positive factor in solid tumor growth. Cancer Res. 2000;60(15): 4010-4015.

8. Yasuda H. Solid tumor physiology and hypoxia-induced chemo/radioresistance: novel strategy for cancer therapy: nitric oxide donor as a therapeutic enhancer. Nitric Oxide. 2008;19(2):205-216.

9. Comerford KM, Wallace TJ, Karhausen J, Louis NA, Montalto MC, Colgan SP. Hypoxia-inducible factor-1-dependent regulation of the multidrug resistance (MDR1) gene. Cancer Res. 2002;62(12):3387-3394.

10. Zheng X, Wang X, Mao H, Wu W, Liu B, Jiang X. Hypoxia-specific ultrasensitive detection of tumours and cancer cells in vivo. Nat Commun. 2015;6(1):5834

11. Zeng Y, Liu Y, Shang J, et al. Phosphorescence monitoring of hypoxic microenvironment in solid-tumors to evaluate chemotherapeutic effects using the hypoxia-sensitive iridium (III) coordination compound. PLoS One. 2015;10(3):e0121293.

12. Zhang S, Hosaka M, Yoshihara T, et al. Phosphorescent light-emitting iridium complexes serve as a hypoxia-sensing probe for tumor imaging in living animals. Cancer Res. 2010;70(11):4490-4498.

13. Aquino-Parsons C, Luo C, Vikse CM, Olive PL. Comparison between the comet assay and the oxygen microelectrode for measurement of tumor hypoxia. Radiother Oncol. 1999;51(2):179-185.

14. Kim IH, Lemmon MJ, Brown JM. The influence of irradiation of the tumor bed on tumor hypoxia: measurements by radiation response, oxygen electrodes, and nitroimidazole binding. Radiat Res. 1993;135(3): 411-417.

15. Minagawa Y, Shizukuishi K, Koike I, et al. Assessment of tumor hypoxia by ${ }^{62} \mathrm{Cu}-\mathrm{ATSM} \mathrm{PET} / \mathrm{CT}$ as a predictor of response in head and neck cancer: a pilot study. Ann Nucl Med. 2011;25(5):339-345.

16. Hoskin PJ, Carnell DM, Taylor NJ, et al. Hypoxia in prostate cancer: correlation of BOLD-MRI with pimonidazole immunohistochemistryinitial observations. Int J Radiat Oncol Biol Phys. 2007;68(4):1065-1071.

17. Knox HJ, Kim TW, Zhu Z, Chan J. Photophysical tuning of $N$-oxidebased probes enables ratiometric photoacoustic imaging of tumor hypoxia. ACS Chem Biol. 2018;13(7):1838-1843.

18. Zhang G, Palmer GM, Dewhirst MW, Fraser CL. A dual-emissivematerials design concept enables tumour hypoxia imaging. Nat Mater. 2009;8(9):747-751.

19. Chinen AB, Guan CM, Ferrer JR, Barnaby SN, Merkel TJ, Mirkin CA. Nanoparticle probes for the detection of cancer biomarkers, cells, and tissues by fluorescence. Chem Rev. 2015;115(19):10530-10574.

20. Freeman TM, Seitz WR. Oxygen probe based on tetrakis (alkylamino) ethylene chemiluminescence. Anal Chem. 1981;53(1):98-102.

21. Ramamoorthy R, Dutta PK, Akbar SA. Oxygen sensors: Materials, methods, designs and applications. J Mater Sci. 2003;38(21):4271-4282.
22. Chu CS, Lo YL. Ratiometric fiber-optic oxygen sensors based on sol-gel matrix doped with metalloporphyrin and 7-amino-4-trifluoromethyl coumarin. Sens Actuators B. 2008;134(2):711-717.

23. Liu J, Wu Y, Yu Y, Li K, Ji Y, Wu D. Quantitative ratiometric phosphorescence hypoxia-sensing nanoprobes based on quantum dots/Ir(III) glycerol monoolein cubic-phase nanoparticles. Biosens Bioelectron. 2017;98:119-125

24. Lv W, Zhang Z, Zhang KY, et al. A mitochondria-targeted photosensitizer showing improved photodynamic therapy effects under hypoxia. Angew Chem Int Ed Engl. 2016;55(34):9947-9951.

25. Ryan HE, Lo J, Johnson RS. HIF-1 alpha is required for solid tumor formation and embryonic vascularization. EMBOJ. 1998;17(11):3005-3015.

26. Huang WC, Shen MY, Chen HH, et al. Monocytic delivery of therapeutic oxygen bubbles for dual-modality treatment of tumor hypoxia. J Control Release. 2015;220(Pt B):738-750.

27. Wang Y, Mirza S, Wu S, et al. 3D hydrogel breast cancer models for studying the effects of hypoxia on epithelial to mesenchymal transition. Oncotarget. 2018;9(63):32191-32203.

28. Zheng X, Tang H, Xie C, Zhang J, Wu W, Jiang X. Tracking cancer metastasis in vivo by using an iridium-based hypoxia-activated optical oxygen nanosensor. Angew Chem Int Ed Engl. 2015;54(28):8094-8099.

29. Servagi-Vernat S, Differding S, Hanin FX, et al. A prospective clinical study of ${ }^{18} \mathrm{~F}$-FAZA PET-CT hypoxia imaging in head and neck squamous cell carcinoma before and during radiation therapy. Eur J Nucl Med Mol Imaging. 2014;41(8):1544-1552.

30. Lee DE, Koo H, Sun IC, Ryu JH, Kim K, Kwon IC. Multifunctional nanoparticles for multimodal imaging and theragnosis. Chem Soc Rev. 2012;41(7):2656-2672.

31. Perfézou M, Turner A, Merkoçi A. Cancer detection using nanoparticlebased sensors. Chem Soc Rev. 2012;41(7):2606-2622.

32. Maeda H, Wu J, Sawa T, Matsumura Y, Hori K. Tumor vascular permeability and the EPR effect in macromolecular therapeutics: a review. J Control Release. 2000;65(1-2):271-284.

33. Sy L, Xie BR, Cheng H. A biomimetic theranostic $\mathrm{O}_{2}$-meter for cancer targeted photodynamic therapy and phosphorescence imaging. Biomaterials. 2018;151:1-12.

34. Ghosh Chaudhuri R, Paria S. Core/shell nanoparticles: classes, properties, synthesis mechanisms, characterization, and applications. Chem Rev. 2012;112(4):2373-2433.

35. Schärtl W. Current directions in core-shell nanoparticle design. Nanoscale. 2010;2(6):829-843.

36. Xu S, Zhang X, Xu H, et al. Silane modified upconversion nanoparticles with multifunctions: imaging, therapy and hypoxia detection. Sci Rep. 2016;6(1):22350.

37. Huang YY, Tian Y, Liu XQ, et al. Luminescent supramolecular polymer nanoparticles for ratiometric hypoxia sensing, imaging and therapy. Mater Chem Front. 2018;2(10):1893-1899.

38. Wu W, Jiang C, Roy VAL. Recent progress in magnetic iron oxidesemiconductor composite nanomaterials as promising photocatalysts. Nanoscale. 2015;7(1):38-58.

39. Yu Y, Wu Y, Liu J, Liu Y, Wu D. Highly efficient dual-modal phosphorescence/computed tomography bioprobes based on an iridium complex and AuNP polyiohexol composite nanoparticles. Nanoscale. 2017;9(27):9447-9456.

40. Kinge S, Crego-Calama M, Reinhoudt DN. Self-assembling nanoparticles at surfaces and interfaces. Chemphyschem. 2008;9(1):20-42.

41. Grzelczak M, Vermant J, Furst EM, Liz-Marzán LM. Directed selfassembly of nanoparticles. ACS Nano. 2010;4(7):3591-3605.

42. Bobo D, Robinson KJ, Islam J, Thurecht KJ, Corrie SR. Nanoparticlebased medicines: a review of FDA-approved materials and clinical trials to date. Pharm Res. 2016;33(10):2373-2387.

43. Zhou X, Liang H, Jiang P, et al. Multifunctional phosphorescent conjugated polymer dots for hypoxia imaging and photodynamic therapy of cancer cells. Adv Sci. 2016;3(2):1500155.

44. Pattni BS, Chupin VV, Torchilin VP. New developments in liposomal drug delivery. Chem Rev. 2015;115(19):10938-10966.

45. Luk BT, Zhang L. Cell membrane-camouflaged nanoparticles for drug delivery. J Control Release. 2015;220(Pt B):600-607. 
46. Xing $\mathrm{H}, \mathrm{Bu} \mathrm{W}$, Zhang S, et al. Multifunctional nanoprobes for upconversion fluorescence, MR and CT trimodal imaging. Biomaterials. 2012; 33(4):1079-1089.

47. Jana A, Crowston BJ, Shewring JR, et al. Heteronuclear Ir(III)-Ln(III) luminescent complexes: small-molecule probes for dual modal imaging and oxygen sensing. Inorg Chem. 2016;55(11):5623-5633.

48. Lucky SS, Soo KC, Zhang Y. Nanoparticles in photodynamic therapy. Chem Rev. 2015;115(4):1990-2042.

49. Wang Z, Ma R, Yan L, Chen X, Zhu G. Combined chemotherapy and photodynamic therapy using a nanohybrid based on layered double hydroxides to conquer cisplatin resistance. Chem Commun. 2015;51(58): 11587-11590.

50. Stich MI, Fischer LH, Wolfbeis OS. Multiple fluorescent chemical sensing and imaging. Chem Soc Rev. 2010;39(8):3102-3114.

51. Robertson CA, Evans DH, Abrahamse H. Photodynamic therapy (PDT): A short review on cellular mechanisms and cancer research applications for PDT. J Photochem Photobiol B. 2009;96(1):1-8.

52. Feng Z, Tao P, Zou L, et al. Hyperbranched phosphorescent conjugated polymer dots with iridium(III) complex as the core for hypoxia imaging and photodynamic therapy. ACS Appl Mater Interfaces. 2017; 9(34):28319-28330.

53. Wang XH, Peng HS, Yang W, Ren ZD, Liu YA. Mitochondria-targeted theranostic nanoparticles for optical sensing of oxygen, photodynamic cancer therapy, and assessment of therapeutic efficacy. Microchimica Acta. 2016;183(10):2723-2731.

54. Lv Z, Zou L, Wei H, Liu S, Huang W, Zhao Q. Phosphorescent starburst $\mathrm{Pt}(\mathrm{II})$ porphyrins as bifunctional therapeutic agents for tumor hypoxia imaging and photodynamic therapy. ACS Appl Mater Interfaces. 2018;10(23):19523-19533.

55. Wang J, Kou J, Hou X, Zhao Z, Chao H. A ruthenium(II) anthraquinone complex as the theranostic agent combining hypoxia imaging and HIF-1 $\alpha$ inhibition. Inorganica Chimica Acta. 2017;454:176-183.

56. Li H, Lei W, Wu J, et al. An upconverting nanotheranostic agent activated by hypoxia combined with NIR irradiation for selective hypoxia imaging and tumour therapy. J Mater Chem B. 2018;6(18):2747-2757.

57. Thambi T, Park JH, Lee DS. Hypoxia-responsive nanocarriers for cancer imaging and therapy: recent approaches and future perspectives. Chem Commun. 2016;52(55):8492-8500.

58. Jain RK, Stylianopoulos T. Delivering nanomedicine to solid tumors. Nat Rev Clin Oncol. 2010;7(11):653-664.

59. Netti PA, Berk DA, Swartz MA, Grodzinsky AJ, Jain RK. Role of extracellular matrix assembly in interstitial transport in solid tumors. Cancer Res. 2000;60(9):2497-2503.

60. Pluen A, Boucher Y, Ramanujan S, et al. Role of tumor-host interactions in interstitial diffusion of macromolecules: Cranial vs subcutaneous tumors. Proc Natl Acad Sci U S A. 2001;98(8):4628-4633.

61. Wong C, Stylianopoulos T, Cui J, et al. Multistage nanoparticle delivery system for deep penetration into tumor tissue. Proc Natl Acad Sci USA. 2011;108(6):2426-2431
62. Xie Z, Guo W, Guo N, et al. Targeting tumor hypoxia with stimulusresponsive nanocarriers in overcoming drug resistance and monitoring anticancer efficacy. Acta Biomater. 2018;71:351-362.

63. Torchilin V. Tumor delivery of macromolecular drugs based on the EPR effect. Adv Drug Deliv Rev. 2011;63(3):131-135.

64. Brown KC. Peptidic tumor targeting agents: the road from phage display peptide selections to clinical applications. Curr Pharm Des. 2010;16(9): 1040-1054.

65. Zhou Z, Wagh NK, Ogbomo SM, et al. Synthesis and in vitro and in vivo evaluation of hypoxia-enhanced ${ }^{111} \mathrm{In}$-bombesin conjugates for prostate cancer imaging. J Nucl Med. 2013;54(9):1605-1612.

66. Torchilin VP, Lukyanov AN. Peptide and protein drug delivery to and into tumors: challenges and solutions. Drug Discov Today. 2003;8(6): 259-266.

67. Veronese FM. Peptide and protein PEGylation: a review of problems and solutions. Biomaterials. 2001;22(5):405-417.

68. Brigger I, Dubernet C, Couvreur P. Nanoparticles in cancer therapy and diagnosis. Adv Drug Delivery Rev. 2012;64(5):24-36.

69. Rao J. Shedding light on tumors using nanoparticles. ACS Nano. 2008;2(10):1984-1986.

70. Schöttler S, Becker G, Winzen S, et al. Protein adsorption is required for stealth effect of poly(ethylene glycol)- and poly(phosphoester)-coated nanocarriers. Nat Nanotechnol. 2016;11(4):372-377.

71. Pansare V, Hejazi S, Faenza W, Prud'homme RK. Review of longwavelength optical and NIR imaging materials: contrast agents, fluorophores and multifunctional nano carriers. Chem Mater. 2012;24(5): $812-827$.

72. Luo S, Zhang E, Su Y, Cheng T, Shi C. A review of NIR dyes in cancer targeting and imaging. Biomaterials. 2011;32(29):7127-7138.

73. Zhou J, Liu Z, Li F. Upconversion nanophosphors for small-animal imaging. Chem Soc Rev. 2012;41(3):1323-1349.

74. Park J, Lee J, Kwag J, et al. Quantum dots in an amphiphilic polyethyleneimine derivative platform for cellular labeling, targeting, gene delivery, and ratiometric oxygen sensing. ACS Nano. 2015;9(6): 6511-6521.

75. Luo $\mathrm{CH}$, Huang $\mathrm{CT}$, Su CH, Yeh CS. Bacteria-mediated hypoxiaspecific delivery of nanoparticles for tumors imaging and therapy. Nano Lett. 2016;16(6):3493-3499.

76. Shi H, Ma X, Zhao Q, et al. Ultrasmall phosphorescent polymer dots for ratiometric oxygen sensing and photodynamic cancer therapy. Adv Funct Mater. 2014;24(30):4823-4830.

77. Qian C, Yu J, Chen Y, et al. Light-activated hypoxia-responsive nanocarriers for enhanced anticancer therapy. Adv Mater. 2016;28(17): 3313-3320.

78. Liu H, Xie Y, Zhang Y, et al. Development of a hypoxia-triggered and hypoxic radiosensitized liposome as a doxorubicin carrier to promote synergetic chemo-/radio-therapy for glioma. Biomaterials. 2017;121: $130-143$.
International Journal of Nanomedicine

\section{Publish your work in this journal}

The International Journal of Nanomedicine is an international, peerreviewed journal focusing on the application of nanotechnology in diagnostics, therapeutics, and drug delivery systems throughout the biomedical field. This journal is indexed on PubMed Central, MedLine, CAS, SciSearch $®$, Current Contents $\AA /$ Clinical Medicine,

\section{Dovepress}

Journal Citation Reports/Science Edition, EMBase, Scopus and the Elsevier Bibliographic databases. The manuscript management system is completely online and includes a very quick and fair peer-review system, which is all easy to use. Visit http://www.dovepress.com/ testimonials.php to read real quotes from published authors. 